\title{
Conference briefings
}

\section{Therapeutic approaches to autism: research and practice*}

\author{
T. P. Berney, Consultant in the Child Psychiatry of Handicap, Fleming Nuffield Child \\ Psychiatry Unit, Newcastle upon Tyne NE2 3AX
}

This, the third in a series of annual conferences, took treatment as its theme, the biological and psychological anomalies of autism being addressed in previous years. As before, the size was limited to about 50 people but, because of the Gulf war, this year's attendance was predominantly British.

A wide-ranging and sometimes rugged variety of treatments are promoted for autism and attract substantial amounts of money from parents and local authorities. Each philosophy has its practitioneradvocate who presents a testimony of success with a religious conviction on the basis of their subjective observation of a small number of cases, selected because they have stayed the course. The Nottingham team gave a particularly helpful overview of the field with a comparative study which included several types of special school as well as Waldon and Holding Therapies. The results have yet to be quantified and this will not be easy as many of the children receive a mixture of therapies in a variety of non-residential settings. Other presentations were of an initial evaluation of Daily Life Therapy from the Boston Higashi School and a Norwegian study of the effectiveness of low casein and gluten diets. These were hampered by their lack of suitable comparisons to control for age-related or spontaneous change.

Reviews of other approaches included the Option Method and low social intrusion teaching as well as

-A conference held at Durham University, from 3 to 6 April 1991, arranged by the National Autistic Society and Autism Europe. some practical strategies to teach social empathy. As in the blind men's description of an elephant, differing views revealed different facets of a complex syndrome. For example, a Canadian account which focused on sensory perceptions suggested that a form of signal overload, the imbalance and volume of auditory or visual input, might contribute to social withdrawal.

Although the therapeutic emphasis was on childhood, the growing concern about adult habilitation was acknowledged in presentations of the Irish and Seaham communities, the latter including a visit. The Irish community, self-sufficient in the monastic tradition, made a contrast with Seaham which leans towards the integrated small staffed house of 'care in the community'. From the resident's viewpoint they have much in common in their emphasis on the acquisition of social and domestic skills.

Such well-publicised choice has two main effects. The first is to leave families wondering whether they have done all that they might; the second is to encourage them to shop on. Most evaluations promote the positive effects of treatment but, in their neglect of the drop-out, overlook any adverse effects either on the child or on its family. The treatment of most psychiatric disorders has moved through a similar scientific dark age, characterised by the uncertain but enthusiastic exploration of a variety of therapies. Autism is no exception and meetings such as this help damage-limitation by giving some sense of proportion.

\section{Hearing voices*}
Frances Klemperer, Research Associate, Department of Psychiatry, United Medical and Dental Schools, Guy's Hospital, London SE1 9RT

This was the second UK conference organised by, and for, people who 'hear voices'. It offered an opportunity for people to meet outside a psychiatric * A national conference organised by Lambeth Link SelfAdvocacy Project, at the International Students' House, Great Portland Street, London, 22 March 1991. environment to discuss their experiences, and promoted the formation of a national network of 'Hearing Voices' self-help groups.

The conference was inspired principally by the work of Professor Marius Romme, Professor of Social Psychiatry, Limburg University, Maastricht, 
The Netherlands. One of Professor Romme's patients (diagnosed as schizophrenic) had felt very isolated by her auditory hallucinations but benefited from developing a theory to account for her experiences. Professor Romme and the patient decided to find others with whom she could share her experiences and her views. They talked on a Dutch television programme, inviting others to join them; 700 people responded, 450 of whom heard voices. They interviewed those who could cope with their 'voices' and selected 20 people who were able and willing to describe their experiences and their coping strategies. They became speakers at a conference for people who hear voices (Romme \& Escher, 1989).

In November 1990 the first UK conference was held in Manchester. Its success led to the establishment of a self-help group and a newsletter and the planning by Lambeth Link of a second conference in London. Channel 4 expressed an interest in filming an 'After Dark' programme on 'hearing voices'. The Independent on Sunday ran an article. Greater London Radio interviewed two of the organisers. Lambeth Link was flooded with calls and received several hundred applications for the conference. They now plan to hold further conferences in London and Manchester.

The morning conference session included presentations by Professor Romme and other professionals involved in research into 'hearing voices'. Alan Leader of Lambeth Link and Ann Walton of the Manchester Hearing Voices Group described their personal experiences, their own interpretations and their coping strategies. The afternoon session was devoted to group discussions, some exclusively for those who heard voices, others also open to professionals.

The conference suggested that hearing voices was a variant of normal experience. Analogies were drawn with left-handedness and homosexuality, once regarded as pathological and subjected to treatment. We were reminded that other cultures, both past and present, sanctioned hearing voices. Healthy people who heard voices did not wish to be regarded as 'ill', or as needing 'treatment', and expressed frustration with medical services which were often unable to assist them, or which offered only stigmatising labels. Some participants clearly valued their voices and made positive use of them. But many also openly acknowledged that hearing voices could be extremely distressing. Some, but by no means all, were helped by medication. There was much interest in sharing coping strategies, with an emphasis on using personal understanding of the experience, for example as the expression of past repressed emotions or as one's conscience, to facilitate coping. Paramount was the need to be regarded, not as a case of pathology, but as an individual, each with his or her unique experience and own understanding of the 'voices'.

Individuals who heard voices valued the chance that this conference offered to share their experiences. The mental health professionals were offered a refreshing view of alternative approaches to hallucinations, and were given the privilege of hearing a wide variety of accounts quite unlike that brought to a typical psychiatric out-patient clinic.

Many psychiatrists would dispute the psychodynamic explanations, and argue that although these may be pathoplastic in hallucinations they are only rarely pathogenic. Although hallucinations may occur as isolated phenomena, they may be associated with neurophysiological pathology and are an important symptom of the syndrome of schizophrenia. But using 'biological' explanations may deter an individual from gaining control over his 'voices'. The strength of this conference was to emphasise that developing a positive understanding of the individual's experience must be a central concern.

Five Hearing Voices self-help groups are now established or being established in Manchester, London, Plymouth, Nottingham and Belfast. As well as providing support and exchanging information, the network has an ambitious aim: to convince not just the medical profession, but also the general public, that it is possible to live an ordinary and 'normal' life, despite having extra-ordinary experiences.

\section{Reference}

Romme, M. A. J. \& Escher, A. D. M. A. C. (1989) Hearing voices. Schizophrenia Bulletin, 15, 209-216.

The Hearing Voices network and newsletter can be contacted through M.C.V.S., Fourways House, 57 Hilton Street, Piccadilly, Manchester MI 2EJ (telephone 0612363206 ).

The London Hearing Voices Group can be contacted through Alan Leader, Lambeth Link Self-Advocacy Project, 30A Acre Lane, Brixton, London SW2 5SG (telephone 071978 8766). 